Original article

\title{
Cost-effectiveness of point-of-care devices for detection of anemia in community settings in India
}

\author{
Sutapa B. Neogi ${ }^{\text {a, }}$, Denny John ${ }^{\mathrm{b}}$, Jyoti Sharma ${ }^{\mathrm{c}}$, Rakhee Kar ${ }^{\mathrm{d}}$, Sitanshu Sekhar Kar ${ }^{\mathrm{d}}$, \\ Maitreyee Bhattacharya ${ }^{\mathrm{e}}$, Kartavya $\operatorname{Tiwari}^{\mathrm{c}}$, Renu Saxena ${ }^{\mathrm{f}}$ \\ ${ }^{a}$ Indian Institute of Public Health Delhi, Currently at International Institute of Health Management Research (IIHMR), Delhi, India \\ ${ }^{\mathrm{b}}$ Campbell Collaboration, New Delhi, India \\ ${ }^{\mathrm{c}}$ Indian Institute of Public Health Delhi, India \\ d JIPMER, Puducherry, India \\ e Calcutta Medical College, Kolkata, India \\ ${ }^{\mathrm{f}}$ AIIMS, New Delhi, India
}

\section{Background}

Anemia is a public health problem affecting $46.3 \%$ of the population in India. ${ }^{1}$ It affects $58 \%$ children under five years of age, $54 \%$ adolescent girls of $15-19$ years of age, $53 \%$ women of reproductive age, $50 \%$ pregnant mothers, and $29 \%$ adolescent boys in the country. ${ }^{2}$ Detection of anemia is important for institution of appropriate management. Several methods are available for diagnosis of anemia especially in outreach and field settings where well-equipped laboratories are unavailable. The devices have been tested in diverse settings and have yielded varied levels of accuracy. ${ }^{3-7}$ In the absence of a concrete evidence, there is a need to examine the devices that have the potential to be included in public health programs. Given the fact that most of the screening happens in community and outreach settings where provision for a laboratory support seems difficult, the device ought to be tested in field settings with health workers (Auxillary Nurse Midwives or ANMs) as end users.

Currently four such devices have been identified that have the potential to be used in public health settings, namely, digital hemoglobinometers (True $\mathrm{Hb}$ and Hemocue), and non-invasive devices, i.e. Masimo Pulse Oximetry test and Spectroscopic spectroscopic device test. ${ }^{8}$ Identification of a suitable device for public health services warrants analysis of parameters other than the diagnostic accuracy. Some such parameters include cost of devices, cost effectiveness, user-friendliness and operational factors.

There is a need to evaluate them in order to identify the most cost effective device suitable for use in Indian and similar settings.
A multicentric study across four sites was undertaken to assess the diagnostic accuracy (effectiveness) of these devices (described elsewhere).$^{9}$ The objective of the current study is to estimate the cost effectiveness of the devices by evaluating the incremental cost for each case detected from the health systems perspective. The secondary objective is to assess the user friendliness and operational issues of the devices for detection of anemia in field settings in India.

\section{Methods}

\subsection{Context}

An analysis of the performance of some such devices was undertaken recently. Four devices (index tests) namely, True Hb Digital Hemoglobinometer, HemoCue, Masimo's non-invasive device and Spectroscopic Spectroscopic devices were examined for diagnostic accuracy on an adequate sample of adult population attending field practice areas of Medical colleges in India: Jawaharlal Institute of Postgraduate Medical Education and Research (JIPMER), Puducherry, and Calcutta Medical College, Kolkata, West Bengal. To test the accuracy in different weather conditions, the devices were additionally tested at All India Institute of Medical Sciences (AIIMS) Jodhpur and Rural hospital, Reckong Peo (Himachal Pradesh). The index tests were compared against autoanalyzer (gold standard). Accuracy was expressed by sensitivity, specificity, likelihood ratios, predictive values and area under the curve (AUC) and levels of agreement. According to this study, HemoCue and TrueHb performed better than Massimo and spectroscopic devices.

\footnotetext{
* Corresponding author.

E-mail addresses: sutapa@iihmrdelhi.edu.in (S.B. Neogi),djohn1976@gmail.com (D. John), jyoti@iiphd.org (J. Sharma), drrakheekar@gmail.com (R. Kar), drsitanshukar@gmail.com (S.S. Kar), mbhattyacharyya@yahoo.co.in (M. Bhattacharya), kartavyatiwari25@gmail.com (K. Tiwari), renusaxena@outlook.com (R. Saxena).
} 
Detection of anemia by technicians was similar between TrueHb and HemoCue (AUC $0.92 \mathrm{v} / \mathrm{s}$ 0.90, p > 0.05). Community workers performed better with Hemocue for detecting anemia compared to TrueHb (AUC $0.92 \mathrm{v} / \mathrm{s} 0.90, \mathrm{p}<0.05$ ). For detection of severe anemia, accuracy of TrueHb was significantly better with technicians (AUC $0.91 \mathrm{v} / \mathrm{s} 0.70$; $\mathrm{p}<0.05$ ) and community workers (AUC $0.91 \mathrm{v} / \mathrm{s} 0.73 ; \mathrm{p}<0.05$ ). HemoCue showed a bias or mean difference (95\%CI) of $0.47 \mathrm{~g} / \mathrm{dl}(0.42$, $0.52)$, and $0.92 \mathrm{~g} / \mathrm{dl}(0.82,1.03)$ for severe anemia. For TrueHb, it was $-0.28 \mathrm{~g} / \mathrm{dl}(-0.37,-0.20)$ for all readings, and $0.06 \mathrm{~g} / \mathrm{dl}(-0.52,0.63)$ for severe anemia. ${ }^{9}$

\subsection{Design}

This study on cost effectiveness was undertaken alongside the diagnostic accuracy study with cross-sectional design. The data collection for the study was conducted between August 2018 and March 2019. The protocol of the cost-effectiveness analysis approach has been published elsewhere. $^{10}$

\subsection{Costs and cost-effectiveness analysis}

The costs were captured through primary data collection using a micro-costing (bottom up) methodology from the study sites. At the first step, every input consumed in the detection of anemia using the pointof-care devices in an individual was identified. Secondly, the quantity of each resource item, such as human resource (ANM time for each test), equipment (device, charger, adapter), accessories (micro cuvettes/ strips), consumables (used in test), non-medical (items in facility rooms), and capital space (i.e. the space used for conducting the tests) was identified. At the third step, unit costs were calculated for each of these resource items. Inputs from device manufacturers were used to cost the equipment and accessories, while procurement costs were considered for consumables and no-medical costs items. Unit cost for ANM was calculated by dividing the total salary per month divided by the time taken by ANM for each test (i.e. 26 working days with $7 \mathrm{~h}$ per day was time considered per month for receiving full salary). Other resource costs was calculated by multiplying the per unit costs with the total units consumed. Rental value for the space used for testing was captured using reference rental values prevalent in the area. Since the study sites were government hospitals, shared costs, and administrative costs were not captured as the administration requirement for receiving this data was time-consuming, hence these costs were not included in the total cost analysis.

Data on costs and sensitivity of the devices for anemia detection were collected simultaneously at each study site.

Effectiveness is defined here as the case detection rate or sensitivity of the devices to detect anemia and severe anemia. The details are available elsewhere. ${ }^{10}$

Cost effectiveness analysis was performed to assess the relative efficiency of the invasive and non-invasive methods for detecting anemia by health workers. The Incremental Cost Effectiveness Ratios (ICERs) were calculated from costs (reported in this study) and effectiveness (reported elsewhere). ${ }^{9}$ The ICER was calculated as: [(Mean cost per test) device A - (Mean cost per test) device B]/[(Detection rate) device A (Detection rate) device B]. The accuracy of the devices was compared against the autoanalyzer (gold standard) in the main study. However, the utility of autoanalyzer and point of care devices are very different and hence comparison for cost effective analysis was difficult. In the absence of a suitable comparator, for the purpose of calculations, we considered HemoCue against which all other devices were compared. Since the data for both costs and effectiveness were for an annual year, the costs were not discounted as per standard guidelines for economic evaluations. $^{11}$

Sensitivity analysis was conducted with lower and upper bounds of sensitivity of diagnostic parameters across all medical devices.

Scenario analysis for detection of anemia in an annual year was also conducted with the assumption that an ANM can screen patients 2 days/ week for 1 year (approximately 50 weeks), and assuming $10 \mathrm{~min}$ for each test, will be able to examine 6 patients in $1 \mathrm{~h}$ or 42 patients per day (assuming $7 \mathrm{~h}$ of work per day). The scenario also assumes that the ANMs will be conducting testing for anemia with no outside work.

The analysis was done from a quasi-health system perspective with a time horizon of one year. The outcome measure was 'correct diagnosis' that referred to the sensitivity of the devices i.e. ability of the devices to identify true positives. Given the fact that severe anemia is a priority of the national program, cost effectiveness was calculated separately for anemia and severe anemia. Reporting of study findings followed CHEERS guidelines. ${ }^{11}$

Although cost utility analysis would have been a better estimate, this was not deemed appropriate in the current study. Hence, it was not included.

\subsection{Additional components}

Operational issues while using a device also aid in taking a decision on the feasibility of its use in field settings. We therefore explored different aspects like ease of use, portability, objectivity in readings, average time taken to perform each test, expertise required and strip/ cuvette wastage. These were gathered from specifications mentioned in product inserts, from the manufacturers and research staff involved in the study. None of the patients had any immediate complications following needle pricks although we could not record any long term effects due to logistic reasons. Laboratory technicians and ANMs independently asked to rate each of the following criteria: ease of use, efficiency in daylight, scope of subjective errors, portability, convenience to patient, interpretation of hemoglobin $(\mathrm{Hb})$ results, need for power/ battery, average time taken for performing one test, and expertise required, on a scale of 1 (very poor) to 5 (excellent) for each device method. The total score across each of these parameters for laboratory technicians and ANMs were used to identify user friendliness for each method (maximum score for each method). These were subjective assessments to understand the perspectives of the users (4 per study site, 16 health workers in total).

\section{Results}

The individual cost of every device is shown in Table 1 . The cost of conducting each test was minimum with Massimo (INR 104.8) in rural areas and with Spectroscopic (INR 140.0) in urban and maximum with TrueHb (INR 137.0 in rural and 177.4 in urban areas). Costs in urban areas are more than in rural areas. Additional cost to the health system for conducting each test in rural areas was found to be INR 24.4 with HemoCue while it was INR 38.7 with TrueHb. (Table 1).

The cost of consumables of testing devices contributed highest among Hemocue $(12.1 \%$ of total cost), and True Hb (11.5\% of total) in urban areas. The non-medical items in the examination room (such as furniture-examination table chairs, cupboards, curtains, wash basins, soaps, buckets, light, fan etc) contributed to the majority of the total costs for each device.

The cost calculated per case detected and effectiveness results (case detection rate or sensitivity) were calculated to compute the ICER for every device. HemoCue was found to be more cost effective than True $\mathrm{Hb}$ for detection of anemia while TrueHb is more cost effective for detection of severe anemia for both urban and rural areas. Since the users in the study were ANMs and we are primarily looking at rural areas where such point of care devices will be better utilized, we present the results of rural areas (Table 2). Sensitivity analysis for upper bound of sensitivity values of diagnostic tests showed higher costs per detection rate.

Table 3 provides results of scenario analysis across all anemia and severe anemia. HemoCue will detect 1869 cases per year per ANM with a cost of INR 224840 per device year, with a wastage of INR 27789.30, for 
Table 1

Costs of resources for each test for measuring Hemoglobin (in INR).

\begin{tabular}{|c|c|c|c|c|c|c|c|c|c|}
\hline \multirow[t]{2}{*}{ Component } & \multirow[t]{2}{*}{ Data sources } & \multicolumn{2}{|l|}{ Hemocue } & \multicolumn{2}{|l|}{ True $\mathrm{Hb}$} & \multicolumn{2}{|c|}{ Spectroscopic device } & \multicolumn{2}{|l|}{ Masimo } \\
\hline & & Rural & Urban & Rural & Urban & Rural & Urban & Rural & Urban \\
\hline Human resource (ANM) & Health systems data & $\begin{array}{l}16.7 \\
(13.9 \%)\end{array}$ & $\begin{array}{l}17.7 \\
(10.7 \%)\end{array}$ & $\begin{array}{l}17.1 \\
(12.5 \%)\end{array}$ & $\begin{array}{l}17.5 \\
(9.9 \%)\end{array}$ & $\begin{array}{l}18.1 \\
(16.5 \%)\end{array}$ & $\begin{array}{l}18.1 \\
(12.9 \%)\end{array}$ & $\begin{array}{l}16.6 \\
(15.8 \%)\end{array}$ & $\begin{array}{l}18.3 \\
(12.6 \%)\end{array}$ \\
\hline $\begin{array}{r}\text { Equipment (device, } \\
\text { charger, adapter) }\end{array}$ & Device manufacturers & $0.2(0.1 \%)$ & $0.2(0.1 \%)$ & $\begin{array}{l}0.004 \\
(0 \%)\end{array}$ & $0.004(0 \%)$ & $\begin{array}{l}0.05 \\
(0.04 \%)\end{array}$ & $\begin{array}{l}0.05 \\
(0.04 \%)\end{array}$ & $3.4(3.2 \%)$ & $3.4(2.3 \%)$ \\
\hline $\begin{array}{l}\text { Accessories (Micro } \\
\text { cuvettes/strips) }\end{array}$ & Device manufacturers & $\begin{array}{l}11.9 \\
(9.9 \%)\end{array}$ & $\begin{array}{l}11.9 \\
(7.2 \%)\end{array}$ & $\begin{array}{l}26.4 \\
(19.3 \%)\end{array}$ & $\begin{array}{l}26.4 \\
(14.9 \%)\end{array}$ & $0.0(0 \%)$ & $0.0(0 \%)$ & $0.0(0 \%)$ & $0.0(0 \%)$ \\
\hline $\begin{array}{l}\text { Consumables (items used } \\
\text { in the test) }\end{array}$ & $\begin{array}{l}\text { Collected from hospital } \\
\text { records }\end{array}$ & $\begin{array}{l}12.3 \\
(10.2 \%)\end{array}$ & $\begin{array}{l}19.7 \\
(12.0 \%)\end{array}$ & $\begin{array}{l}12.3 \\
(9.0 \%)\end{array}$ & $\begin{array}{l}19.7 \\
(11.1 \%)\end{array}$ & $6.1(5.6 \%)$ & $4.3(3.1 \%)$ & $6.1(5.8 \%)$ & $4.3(3.0 \%)$ \\
\hline $\begin{array}{l}\text { Non-medical (items in } \\
\text { facility rooms) }\end{array}$ & $\begin{array}{l}\text { Collected from hospital } \\
\text { records }\end{array}$ & $\begin{array}{l}78.8 \\
(65.5 \%)\end{array}$ & $\begin{array}{l}113.4 \\
(69.0 \%)\end{array}$ & $\begin{array}{l}80.8 \\
(59.0 \%)\end{array}$ & $\begin{array}{l}112.3 \\
(63.3 \%)\end{array}$ & $\begin{array}{l}85.4 \\
(77.6 \%)\end{array}$ & $\begin{array}{l}116.4 \\
(82.9 \%)\end{array}$ & $\begin{array}{l}78.3 \\
(74.7 \%)\end{array}$ & $\begin{array}{l}117.2 \\
(81.0 \%)\end{array}$ \\
\hline Capital space (rental) & $\begin{array}{l}\text { Collected from hospital } \\
\text { records }\end{array}$ & $0.4(0.3 \%)$ & $1.6(0.9 \%)$ & $0.4(0.3 \%)$ & $1.5(0.9 \%)$ & $0.5(0.4 \%)$ & $1.6(1.1 \%)$ & $0.4(0.4 \%)$ & $1.6(1.1 \%)$ \\
\hline Total cost/test (INR) & & 120.3 & 164.3 & 137.0 & 177.4 & 110.1 & 140.4 & 104.8 & 144.7 \\
\hline
\end{tabular}

Table 2

Incremental cost effectiveness ratios (ICER) of devices for detection of anemia and severe anemia (costs in INR).

\begin{tabular}{|c|c|c|c|c|}
\hline Components & HemoCue & TrueHb & $\begin{array}{l}\text { Spectroscopic } \\
\text { device }\end{array}$ & Massimo \\
\hline \multicolumn{5}{|l|}{ Anemia } \\
\hline Base case & Reference & -464.3 & 30.4 & 65.1 \\
\hline $\begin{array}{l}\text { Lower bound of } \\
\text { sensitivity }\end{array}$ & Reference & -407.7 & 28.0 & 60.0 \\
\hline $\begin{array}{l}\text { Upper bound of } \\
\text { sensitivity }\end{array}$ & Reference & -557.1 & 34.0 & 73.3 \\
\hline \multicolumn{5}{|l|}{ Severe anemia } \\
\hline Base case & Reference & 41.5 & 54.1 & 52.2 \\
\hline $\begin{array}{l}\text { Lower bound of } \\
\text { sensitivity }\end{array}$ & Reference & 46.2 & 46.4 & 58.9 \\
\hline $\begin{array}{l}\text { Upper bound of } \\
\text { sensitivity }\end{array}$ & Reference & 45.8 & 96.8 & 53.4 \\
\hline
\end{tabular}

all anemia. In comparison TrueHb will identify 1806 cases with an cost of INR 247422 per device per year, with a wastage of INR 402278. With regards to severe anemia, TrueHb will detected 73 cases with cost of detection at INR 11508 per year. While HemoCue will be able to detect only 39 cases of severe anemia with cost of detection at INR 10105 per year.

While choosing a device for programmatic use, several parameters were taken into consideration. Although accuracy across the spectrum of the condition (anemia in our case) gains maximum weightage, other factors also influence this decision. Of the four devices, HemoCue was rated the best by the health workers followed by TrueHb, Massimo and Spectroscopic device (Table 4). HemoCue fared better in terms of user friendliness, wastage per test, shelf life of the equipment and the accessories, and number of tests that can be done in field settings with fully charged batteries. On the other hand, True $\mathrm{Hb}$ outweighed HemoCue with regards to portability of the device, range of temperature for its use and storage, and feasibility to use it in community settings without having to use batteries (Table 5). Besides, HemoCue showed a bias or mean difference of $0.92 \mathrm{~g} / \mathrm{dl}(0.82,1.03)$ for severe anemia. ${ }^{9}$ Overestimation by nearly $1 \mathrm{gm} \%$ may change the category of anemia and this can influence subsequent management.

\section{Discussion}

The analysis of the four devices shows that HemoCue is more cost effective than TrueHb for detection of anemia whereas True $\mathrm{Hb}$ is more cost effective for severe anemia. The HemoCue was found to be more user friendly than TrueHb as mentioned the health workers. With regards to the operational issues, TrueHb outweighed HemoCue for most factors.

Clinical benefit is expressed not only in terms of quality of life but using diagnostic yield. Economic evaluation of diagnostic tests is different from that of drugs or interventions. ${ }^{12}$ Rapid technology upgradation very often does not allow evaluation of clinical effectiveness. ${ }^{13,14}$ In our case, outcome of anemia is linked to treatment apart from diagnosis that cannot be disaggregated. Moreover, efficacy of the device depends on the users and costs of the devices may vary considerably depending on the numbers of users. There are reports on the possibility of use of Multiple Criteria Decision Analysis (MCDA) to address these challenges, but there is still no definite solution to overcome these with CEA of diagnostic devices. ${ }^{15}$

In a similar study from Malawi, HemoCue was compared to different methods for estimation of $\mathrm{Hb} .{ }^{16}$ Based on accuracy, user friendliness and simplicity, HemoCue was recommended for use in district hospitals. While the authors in that study used accuracy for detection of anemia, we have used accuracy for anemia and severe anemia separately, keeping in mind the requirement of the health system in the country. Supplies and accessories contributed maximally to the cost of an individual test in Malawi while in our case non-medical costs in health facilities had the greatest contribution.

The desirable features that should be highlighted while identifying a device for a national program are accuracy across different field situations in the absence of recalibration and user-friendliness. ${ }^{17}$ We could assess the user-friendliness with health workers as the end users. We also assessed the accuracy across different field situations. However, the devices provided to us were new and the manufacturers mentioned that recalibration of digital hemoglobinometers was not required. We are not quite certain about the requirement of recalibration and therefore this remains a big limitation.

We were not able to calculate the full cost structure due to limited data on costs of training, differential costs and cost of delivering the test. Moreover, the study was done on adult patients for ascertainment of accuracy. The national program deals primarily with anemia in pregnant women and children. Any future economic evaluations, if at all done should be able to calculated for population group comprising of pregnant women and children.

This study draws both cost and effectiveness data from a primary study conducted in a low and middle income country (LMIC), i.e. India, which offers strength to this analysis. This is particularly important because evidence from higher economies may tend to focus on most effective solutions regardless of cost. ${ }^{17}$ Diagnostic accuracy tends to vary with the prevalence of the underlying disease condition and hence it is best ascertained in a place where it is likely to be used in future. ${ }^{18}$ Technology development is an iterative process that relies heavily on the experiences of the users, transparent communication between all concerned, and efforts to create an enabling environment best suited for a particular market. ${ }^{19}$ 
Table 3

Scenario analysis for detection of anemia in year using point of care devices.

\begin{tabular}{|c|c|c|c|c|}
\hline & $\begin{array}{l}\text { Haemo } \\
\text { Cue }\end{array}$ & True $\mathrm{Hb}$ & $\begin{array}{l}\text { Spectroscopic } \\
\text { device }\end{array}$ & Masimo \\
\hline \multicolumn{5}{|l|}{ Detection of anemia } \\
\hline $\begin{array}{l}\text { Total Cost Per unit Test } \\
\text { (INR) }\end{array}$ & 120.3 & 137 & 110.1 & 104.8 \\
\hline $\begin{array}{l}\text { Total number of tests in } \\
1 \text { year per ANM } \\
\text { (approx) }^{\mathrm{a}}\end{array}$ & 4200 & 4200 & 4200 & 4200 \\
\hline $\begin{array}{l}\text { Expected number of } \\
\text { cases of anemia } \\
@ 50 \% \text { prevalence }\end{array}$ & 2100 & 2100 & 2100 & 2100 \\
\hline $\begin{array}{l}\text { Total number of cases of } \\
\text { anemia (true positives } \\
\text { based on sensitivity } \\
\text { data) detected per test }\end{array}$ & 0.89 & 0.86 & 0.56 & 0.66 \\
\hline $\begin{array}{l}\text { Total number of anemia } \\
\text { cases detected per } \\
\text { year }\end{array}$ & 1869 & 1806 & 1176 & 1386 \\
\hline $\begin{array}{l}\text { Cost for detection of } \\
\text { anemia using the } \\
\text { device per year (INR) }\end{array}$ & 224840.7 & 247422 & 129477.6 & 145252.8 \\
\hline $\begin{array}{l}\text { Total cost for the system } \\
\text { for detection of } \\
\text { anemia per year (INR) }\end{array}$ & 252630 & 287700 & 231210 & 220080 \\
\hline $\begin{array}{l}\text { Wastage to the system } \\
\text { (INR) }\end{array}$ & 27789.3 & 40278 & 101732.4 & 74827.2 \\
\hline \multicolumn{5}{|c|}{ Detection of severe anemia } \\
\hline $\begin{array}{l}\text { Total Cost Per unit Test } \\
\text { (INR) }\end{array}$ & 120.3 & 137 & 110.1 & 104.8 \\
\hline $\begin{array}{l}\text { Total number of tests in } \\
1 \text { year per ANM }\end{array}$ & 4200 & 4200 & 4200 & 4200 \\
\hline $\begin{array}{l}\text { Expected number of } \\
\text { cases of severe anemia } \\
@ 2 \% \text { prevalence } \\
\text { (based on available } \\
\text { estimates eg NFHS) }\end{array}$ & 84 & 84 & 84 & 84 \\
\hline $\begin{array}{l}\text { Total number of cases of } \\
\text { severe anemia } \\
\text { detected per test }\end{array}$ & 0.47 & 0.87 & 0.28 & 0.17 \\
\hline $\begin{array}{l}\text { Total number of cases } \\
\text { detected per year }\end{array}$ & 39.48 & 73.08 & 23.52 & 14.28 \\
\hline $\begin{array}{l}\text { Cost for detection of } \\
\text { severe anemia using } \\
\text { the device per year } \\
\text { (INR) }\end{array}$ & 4749.44 & 10011.96 & 2589.55 & 1496.54 \\
\hline $\begin{array}{l}\text { Wastage to the system } \\
\text { (INR) }\end{array}$ & 5355.75 & 1496.04 & 6658.84 & 7306.65 \\
\hline $\begin{array}{l}\text { Total cost for the system } \\
\text { for detection of } \\
\text { anemia per year (INR) }\end{array}$ & 10105.2 & 11508 & 9248.4 & 8803.2 \\
\hline
\end{tabular}

a The total numbers of tests an ANM is required to perform was estimated from a population of 7000-8000 that she has to cater to. The approximate number of pregnant women/@ crude birth rate of 20/1000 results in 150-180 pregnant women and $\mathrm{Hb}$ estimation at every visit makes it (1000 tests/yr) plus nonpregnant women (1000/yr); Adolescents @15\% of population (1100/yr) and @15\% of population children (1100/yr) plus some wastage. Assuming that an ANM can perform 15-20 tests/days for 5-6 days a week thoughout the year, the approximate number of tests per year was assumed to be 4200 .

\section{Conclusion}

To conclude, based on the diagnostic accuracy of the devices across different field conditions, costs and detection rate, for anemia and severe anemia, digital hemoglobinometers performed better than non-invasive devices. Among the versions of digital hemoglobinometers evaluated, we found that for detection of anemia, Hemocue is most cost effective. However, for detection of severe anemia (which is directly related to mortality and other complications), TrueHb was found to be more cost effective. With the evolution of technology, a detailed HTA will be required for re assessment of newer versions of the devices and to revisit the decision.
Table 4

An assessment of user-friendliness of the devices by health workers

$1=$ Poor, $2=$ Below average, $3=$ Average, $4=$ Above Average, $5=$ Excellent.

\begin{tabular}{lllll}
\hline Parameters & HemoCue & TrueHb & $\begin{array}{l}\text { Spectroscopic } \\
\text { device }\end{array}$ & $\begin{array}{l}\text { Massimo } \\
\text { device }\end{array}$ \\
\hline Ease of Use & 4 & 4 & 3 & 4 \\
Efficiency in daylight & 4 & 5 & 3 & 4 \\
Portability & 5 & 5 & 2 & 4 \\
Convenience to Patient & 5 & 4 & 2 & 5 \\
$\quad$ Need for Power/ & 5 & 5 & 2 & 4 \\
$\quad$ Battery & & 3 & 3 & 4 \\
Average time taken for & 5 & & & 4 \\
$\quad$ performing one test & & 4 & 2 & 29 \\
Expertise required & 5 & 30 & 17 & \\
Total Score (out of 35) & 33 & & &
\end{tabular}

Table 5

Features of digital hemoglobinometers (HemoCue and TrueHb).

\begin{tabular}{|c|c|c|}
\hline Parameters & Hemocue & True $\mathrm{Hb}$ \\
\hline $\begin{array}{l}\text { Accuracy (all categories; Case } \\
\text { detection rate or sensitivity }\end{array}$ & $+++(90 \%)$ & $+++(86 \%)$ \\
\hline $\begin{array}{l}\text { Mean diff in Hb compared to } \\
\text { Gold standard for all } \\
\text { categories }\end{array}$ & $+++(0.18)$ & $+++(-0.22)$ \\
\hline $\begin{array}{l}\text { Accuracy (Severe Anemia).. } \\
\text { Case detection rate or } \\
\text { sensitivity }\end{array}$ & - $(47 \%)$ & $+++(87 \%)$ \\
\hline $\begin{array}{l}\text { Mean diff in } \mathrm{Hb} \text { compared to } \\
\text { Gold standard in severe } \\
\text { anemia }\end{array}$ & $-(0.84)$ & $+++(-0.06)$ \\
\hline $\begin{array}{l}\text { Accuracy at different weather } \\
\text { conditions }\end{array}$ & \pm & + \\
\hline Data storage & - (no data) & + (only $\mathrm{Hb}$ values) \\
\hline $\begin{array}{l}\text { Portability (weight of the } \\
\text { device) }\end{array}$ & $+(500 \mathrm{gms})$ & $+++(60 \mathrm{gms})$ \\
\hline $\begin{array}{l}\text { Portability (dimensions) (in } \\
\text { inches) }\end{array}$ & $+(5.5 \times 2.7 \times 6.2)$ & $+++(4.7 \times 2.3 \times 0.3)$ \\
\hline $\begin{array}{l}\text { Ease of use by health workers } \\
\text { (user friendliness) }\end{array}$ & +++ & ++ \\
\hline Ease and duration of training & $+++(4-5 \mathrm{~h})$ & $+++(4-5 \mathrm{~h})$ \\
\hline Objectivity in readings & +++ & +++ \\
\hline Patient convenience & +++ & +++ \\
\hline $\begin{array}{l}\text { Need for recalibration (NR- } \\
\text { Not required) }\end{array}$ & $+++(\mathrm{NR})$ & $+++(\mathrm{NR})$ \\
\hline $\begin{array}{l}\text { Strip/cuvette wastage per test } \\
\text { (Number per test) }\end{array}$ & $++(1.2)$ & $+(1.35)$ \\
\hline Operating temperature & $+\left(10-40^{\circ} \mathrm{C}\right)$ & $++\left(5-45^{\circ} \mathrm{C}\right)$ \\
\hline $\begin{array}{l}\text { Strip/Cuvettes Storage } \\
\text { conditions }\end{array}$ & $+\left(10-40^{\circ} \mathrm{C}\right)$ & $++\left(5-45^{\circ} \mathrm{C}\right)$ \\
\hline Shelf life of the equipment & $+++(7$ years $)$ & $++(5$ years $)$ \\
\hline $\begin{array}{l}\text { Shelf life of strips/ } \\
\text { microcuvettes }\end{array}$ & $++(2$ years $)$ & $+(1$ year $)$ \\
\hline Source of power in facility & ++ (AC adapter) & $++($ Charger $)$ \\
\hline $\begin{array}{l}\text { Source of power in } \\
\text { community }\end{array}$ & $+(4$ AA batteries $)$ & $\begin{array}{l}++ \text { (device should be fully } \\
\text { charged, no batteries } \\
\text { needed }) \text { ) }\end{array}$ \\
\hline $\begin{array}{l}\text { Number of tests in field } \\
\text { settings (fully charged/ } \\
\text { batteries) }\end{array}$ & $++(510)$ & $+(200)$ \\
\hline $\begin{array}{l}\text { Biomedical waste } \\
\text { management }\end{array}$ & $\begin{array}{l}\text { Lancet and } \\
\text { microcuvettes }\end{array}$ & Lancet and strips \\
\hline Approvals & $\begin{array}{l}\text { FDA approved, } \\
\text { CLIA compliant }\end{array}$ & $\begin{array}{l}\text { CLIA compliant, ISO } \\
\text { approved, FDA under } \\
\text { process }\end{array}$ \\
\hline
\end{tabular}

+++ Very good, ++ Good, +Average, - Poor, \pm Equivocal.

\section{Funding}

Department of Health Research [Health Technology Assessment in India (HTAIn) division], Ministry of Health and Family Welfare, Government of India (Sanction letter F.NO.S.11011/02/2017- HR). The funders had no role in designing the study, developing the protocol, data analysis and interpretation and writing the manuscript. 


\section{Declaration of competing interest}

All the authors declare that they do not have conflict of interest.

\section{References}

1 Didzun O, De Neve J-W, Awasthi A, et al. Anaemia among men in India: a nationally representative cross-sectional study. Lancet Global Health. 2019;7(12):e1685-e1694.

2 IIPS: National Family Health Survey 4. in: India Fact Sheet. India: Sciences IIoP. Mumbai; 2015-16.

3 Chaudhary R, Dubey A, Sonker A. Techniques used for the screening of hemoglobin levels in blood donors: current insights and future directions. Hematol Res Rev. 2017; 8:75-88.

4 Dubey A, Murti S. Validation of a hemoglobinometer for use in outdoor blood donation camps. Asian J Transfus Sci. 2016;10(2).

5 Gamal M, Abdelhamid B, Zakaria D, et al. Evaluation of noninvasive hemoglobin monitoring in trauma patients with low hemoglobin levels. Shock: Inj Inflamm Sepsis: Lab Clin Approaches. 2018;49(2):150-153.

6 Neogi SB, Negandhi H, Kar R, et al. Diagnostic accuracy of haemoglobin colour strip (HCS-HLL), a digital haemoglobinometer (TrueHb) and a non-invasive device (TouchHb) for screening patients with anaemia. J clin Pathol. 2016;69(2):164-170.

7 Parker M, Han Z, Abu-Haydar E, et al. An evaluation of hemoglobin measurement tools and their accuracy and reliability when screening for child anemia in Rwanda: randomized study. PLoS One. 2018;13(1). e0187663-e0187663.

8 Neogi S, Negandhi H, Sharma J, Ray S, Saxena R. Diagnostic efficacy of digital hemoglobinometer (TrueHb), HemoCue and non invasive devices for screening patients for anemia in the field settings-a proposal. Indian J Community Health. 2018; 30:86-88. Supp.
9 Neogi SB, Sharma J, Pandey S, et al. Diagnostic accuracy of point-of-care devices for detection of anemia in community settings in India. BMC Health Serv Res. 2020;20(1): 468.

10 Neogi S, John D, Sharma J, et al. Cost-effectiveness of invasive devices versus noninvasive devices for screening of anemia in field settings in India: a study protocol [version 1; peer review: 1 not approved]. F1000Research. 2019;8(861).

11 Husereau D, Drummond M, Petrou S, et al. Consolidated health economic evaluation reporting standards (CHEERS) statement. BMJ Br Med J (Clin Res Ed). 2013;346: f1049.

12 Shinkins B, Yang Y, Abel L, Fanshawe TR. Evidence synthesis to inform model-based cost-effectiveness evaluations of diagnostic tests: a methodological review of health technology assessments. BMC Med Res Methodol. 2017;17(1):56.

13 Drummond M, Griffin A, Tarricone R. Economic evaluation for devices and drugssame or different? Value Health : J Int Soc Pharmacoecon Outcomes Res. 2009;12(4): 402-404.

14 Koffijberg H, van Zaane B, Moons KGM. From accuracy to patient outcome and costeffectiveness evaluations of diagnostic tests and biomarkers: an exemplary modelling study. BMC Med Res Methodol. 2013;13(1):12.

15 Ivlev I, Kneppo P, Bartak M. Multicriteria decision analysis: a multifaceted approach to medical equipment management. Technol Econ Dev Econ. 2014;20(3):576-589.

16 Medina Lara A, Mundy C, Kandulu J, Chisuwo L, Bates I. Evaluation and costs of different haemoglobin methods for use in district hospitals in Malawi. J clin Pathol. 2005;58(1):56-60.

17 McLaren ZM, Sharp A, Hessburg JP, et al. Cost effectiveness of medical devices to diagnose pre-eclampsia in low-resource settings. Dev Eng. 2017;2:99-106.

18 Leeflang MM, Bossuyt PM, Irwig L. Diagnostic test accuracy may vary with prevalence: implications for evidence-based diagnosis. J Clin Epidemiol. 2009;62(1): $5-12$.

19 Kimani FW, Mwangi SM, Kwasa BJ, et al. Rethinking the design of low-cost point-ofcare diagnostic devices. Micromachines (Basel). 2017;8(11):317. 